11,12

\title{
Влияние флуктуаций на образование выделений вторых фаз на границах зерен
}

\author{
(C) П.Е. ЛЬвов ${ }^{1}$, В.В. Светухин ${ }^{1,2}$ \\ ${ }^{1}$ Ульяновский государственный университет, \\ Ульяновск, Россия \\ ${ }^{2}$ Институт нанотехнологий микроэлектроники РАН, \\ Москва, Россия \\ E-mail: LvovPE@Sv.uven.ru
}

(Поступила в Редакцию 13 августа 2018 г. В окончательной редакции 19 сентября 2018 г.)

На основе метода функционала плотности свободной энергии (модифицированного уравнения Кана-Хилларда-Кука) рассмотрена кинетика образования выделений вторых фаз в бинарных сплавах при наличии флуктуаций состава и с учетом влияния границ зерен. Установлено, что на ранней стадии фазового перехода наличие границ зерен и флуктуаций может приводить к возникновению аномальных скоростей роста среднего размера выделений, обусловленных сочетанием различных механизмов распада.

Работа подготовлена в рамках выполнения государственного задания Минобрнауки России (№ 3.2111.2017/4.6) и проекта РФФИ - Правительство Ульяновской области № 16-42-732113.

DOI: $10.21883 /$ FTT.2019.02.47138.232

\section{1. Введение}

Большинство твердых растворов на основе металлов имеют поликристаллическую структуру, то есть состоят из большого числа зерен, имеющих различную ориентацию друг относительно друга [1-5]. Области сопряжения соседних зерен (границы зерен) представляют собой дефекты кристаллической структуры, которые могут оказывать существенное влияние на распределение точечных дефектов и легирующих компонентов сплава, формирование вторых фаз в процессе распада твердого раствора, а также на механические и другие свойства [1-5], которые имеют важное значение для практического применения этого вида материалов.

Одной из важных проблем изучения свойств поликристаллических материалов является задача об установлении влияния границ зерен на процесс фазового перехода первого рода, связанного с формированием выделений вторых фаз [1]. Основным методом анализа данного вида фазовых переходов является метод функционала плотности свободной энергии [6-8], который также может быть применен для анализа формирования выделений на границах раздела фаз $[9,10]$ или на границах зерен [1]. В данном виде фазовых переходов (фазовые переходы смачивания), как правило, рассматривается процесс формирования или трансформации слоя вещества, находящегося на границах зерен $[1,14]$ или подложки [9-13]. При этом смачивающая фаза может быть как жидкой, так и твердой $[1,9,10,14]$, а само смачивание может быть как полным, так и частичным [1,9-14]. В случае частичного смачивания трансформация поверхностного слоя может протекать по спинодальному механизму [10-13] или по механизму нуклеации с формированием уеди- ненных выделений на соответствующих границах раздела [10-14]. Существование смачивающих фазовых переходов на границах зерен наблюдается экспериментально для различных металлических сплавов [14-16].

В работах $[17,18]$ на основе метода функционала плотности свободной энергии была разработана феноменологическая модель, описывающая влияние границ зерен на процесс распределения компонентов между зернограничной областью и объемом зерна. На основе проведенного в $[17,18]$ моделирования показано, что в зависимости от степени пересыщения, температуры сплава, а также характера изменения параметров взаимодействия вблизи границ зерен могут наблюдаться различные типы распределения компонентов системы: обеднение или обогащение зернограничной области, преимущественная зернограничная или объемная преципитация, а также конкурентные и смешанные режимы формирования вторых фаз. С помощью данной модели удается также рассматривать формирование вблизи границ зерен зон, свободных от выделений. Возникновение полного или частичного смачивания границы зерна может наблюдаться при уменьшении параметра взаимодействия вблизи границ зерен [17].

Флуктуации являются одним из наиболее существенных факторов в формировании выделений вторых фаз в области стабильных и метастабильных состояний, где для образования устойчивых зародышей требуется преодоление барьера нуклеации [5]. Существенное влияние флуктуации оказывают как на фазовые переходы первого рода в системах, не содержащих структурных дефектов [19-23], так и на смачивающие фазовые переходы [13]. В связи с этим в данной работе ставится задача обобщения разработанной ранее детерминиро- 
ванной модели $[17,18]$ на случай наличия термических флуктуаций, описываемых в рамках флуктуационнодиссипационной теоремы [22-26]. При этом необходимо установить особенности формирования выделений вторых фаз в условиях флуктуаций.

\section{2. Основные приближения модели}

Рассмотрим на основе метода функционала плотности свободной энергии образование выделений второй фазы в трехмерном фрагменте бинарного сплава кубической формы, имеющем линейный размер L. Пусть внутри рассматриваемого объема расположена плоская неподвижная граница двух зерен, описываемая уравнением $z=L / 2$. В соответствии с предположениями [17] будем считать, что параметр квазихимического взаимодействия $\Omega$ зависит от координат $\Omega=\Omega(x, y, z)$ и описывается экспоненциальной зависимостью вида [18]:

$$
\Omega(x, y, z)=\Omega_{0}\left(1+\Delta \exp \left[-\frac{|z-L / 2|}{\delta_{0}}\right]\right),
$$

где $\Delta=\left(\Omega_{\mathrm{GB}}-\Omega_{0}\right) / \Omega_{0}-$ параметр, определяющий максимальное различие между параметрами взаимодействия в объеме $\left(\Omega_{0}\right)$ и на границе зерна $\left(\Omega_{\mathrm{GB}}\right)$. Изменение параметра взаимодействия $\Omega$ происходит на малой характерной длине $\delta_{0}$, которая составляет величину порядка нескольких периодов решетки $\left(\delta_{0} \ll L\right)$. Знак и величина параметра $\Delta$ влияют на характер распределения компонентов раствора между зернограничной областью и объемом зерна $[17,18]$. В данной работе (для определенности) будем рассматривать случай уменьшения параметра квазихимического взаимодействия по мере приближения к границе зерна $\Delta<0$, что соответствует эффективному уменьшению критической температуры и степени пересыщения вблизи границ зерен [17]. Случай $\Delta>0$ в отсутствие флуктуаций рассматривался в работе [18].

Наличие зависимости параметра взаимодействия от концентрационного поля $c$, а также эффекты, связанные с изменением размера зерна $[27,28]$ или переходом границы зерна в жидкое состояние [1], в данной работе не рассматриваются.

Используя стандартные методы теории функционала плотности свободной энергии [6-8], по аналогии c $[17,18]$ может быть получено модифицированное уравнение Кана-Хилларда-Кука, учитывающее зависимость параметров взаимодействия от координат:

$$
\frac{\partial c}{\partial t}=\nabla\left[M \cdot \nabla\left(\frac{\partial g}{\partial c}-\nabla(\kappa \nabla c)\right)\right]+\sqrt{\varepsilon} \xi,
$$

где $c \equiv c(x, y, z)$ - поле концентрации растворенного компонента, $M-$ подвижность, $\kappa-$ коэффициент градиентной энергии, который связан с параметром квазихимического взаимодействия $\kappa=\kappa_{0} \Omega(x, y, z) a^{2}[17,18]$, $a$ - период решетки, $\kappa_{0}-$ постоянная величина, зависящая от потенциала межатомного взаимодействия компонентов раствора, $\xi$ - случайное гауссовское поле, корреляционная функция для которого описывается с помощью флуктуационно-диссипационной теоремы для сохраняющегося параметра порядка $[25,26]$ :

$$
\left\langle\xi(\mathbf{r}, t) \xi\left(\mathbf{r}^{\prime}, t^{\prime}\right)\right\rangle=-2 M k_{\mathrm{B}} T \nabla^{2} \delta\left(\mathbf{r}-\mathbf{r}^{\prime}\right) \delta\left(t-t^{\prime}\right),
$$

$\varepsilon$ - безразмерная величина, определяющая амплитуду флуктуаций, $\delta$ - дельта-функция Дирака, $k_{\mathrm{B}}-$ постоянная Больцмана, $g \equiv g(c)-$ свободная энергия смешения сплава в расчете на одну частицу в приближении регулярного раствора

$$
g(c)=\Omega c(1-c)+k_{\mathrm{B}} T[c \ln c+(1-c) \ln (1-c)] .
$$

Для сплава, не имеющего структурных дефектов, параметр квазихимического взаимодействия связан с критической температурой $\Omega=2 k_{\mathrm{B}} T_{C}$.

В теории функционала плотности свободной энергии перемещение атомов может быть охарактеризовано эффективным коэффициентом диффузии [7]: $D_{\text {eff }}=\left.M \frac{\partial^{2} g}{\partial c^{2}}\right|_{c_{M}}$. В рассматриваемом случае $(\Delta<0)$ отношение эффективных коэффициентов диффузии на границе и в объеме зерна для среднего состава $c_{M}$ : $D_{\text {eff }}^{b} / D_{\text {eff }}^{v}>1$, что соответствует ускоренной диффузии по границам зерен. Данное отношение становится значительным $\left(D_{\mathrm{eff}}^{b} / D_{\mathrm{eff}}^{v} \gg 1\right)$ только при приближении к границе метастабильности, где вторая производная обращается в нуль. Вместе с тем для ряда сплавов на практике наблюдается существенное (на несколько порядков) повышение коэффициента диффузии на границах зерен (см. например, [29-32]), которое, по всей видимости, связано с уменьшением энергии активации диффузии. Моделирование эволюции таких сплавов может быть выполнено на основе уравнения Кана-Хилларда (или Кана-Хилларда-Кука) с учетом переменной подвижности (см. [33,34]), которую следует принять величиной, зависящей от координат, по аналогии с рассматриваемой в данной модели зависимостью параметра взаимодействия $\Omega[17,18]$. Системы с наличием изменения коэффициента подвижности компонентов сплава вблизи границ зерен в данной работе не рассматриваются.

Переходя в уравнениях (2) и (3) к параметру порядка $\eta=2 c-1$ и считая подвижность $M$ постоянной величиной, преобразуем уравнение (2) к виду:

$$
\frac{\partial \eta}{\partial \tau}=\nabla^{* 2}\left[\phi-\nabla^{*}\left(\kappa^{*} \nabla^{*} \eta\right)\right]+\xi^{*},
$$

где $\quad \kappa^{*}=\frac{\kappa}{\Omega_{0} a^{2}}, \quad \tau=t \frac{M \Omega_{0}}{a^{2}}, \quad \mathbf{r}^{*}=\mathbf{r} / a, \quad \nabla^{*}=a \nabla$, $\xi^{*}=\xi \frac{2 a^{2} \sqrt{\varepsilon}}{m \Omega_{0}} a-$ период решетки, $\phi-$ безразмерная функция имеющая вид

$$
\phi \equiv-2 \Omega^{*} \eta+T^{*} \ln \left[\frac{1+\eta}{1-\eta}\right],
$$

где введены обозначения безразмерных величин $T^{*}=2 k_{\mathrm{B}} T / \Omega_{0}, \Omega^{*}=\Omega(x, y, z) / \Omega_{0}$. Случайное гауссово 
поле $\xi^{*}$ при этом соответствует корреляционной функции вида:

$$
\left\langle\xi^{*}\left(\mathbf{r}^{*}, \tau\right) \xi^{*}\left(\mathbf{r}^{*}, \tau^{\prime}\right)\right\rangle=-4 \varepsilon T^{*} a^{-d} \nabla^{* 2} \delta\left(\mathbf{r}^{*}-\mathbf{r}^{* \prime}\right) \delta\left(\tau-\tau^{\prime}\right),
$$

где $d$ - размерность системы $(d=3)$.

Граничные условия для рассматриваемого фрагмента сплава будем считать периодическими. Начальное условие будем определять случайным гауссовским распределением параметра порядка, характеризуемым средним значением, соответствующим составу сплава $c_{M}$, и малой дисперсией $\sim 10^{-2}$.

\section{3. Численные методы}

Решение уравнения (4) может быть найдено на основе спектрального метода [33-36], который с учетом зависимости параметров взаимодействия от координат $[17,18]$ и наличия флуктуационной составляющей [23-26] приводит к разностной схеме в виде

$$
\begin{aligned}
& \hat{\eta}_{\mathbf{k}}^{n+1}=\hat{\eta}_{\mathbf{k}}^{n}+\frac{1}{1+\Delta \tau \kappa_{0} \tilde{\Omega}|\mathbf{k}|^{4}} \\
& \quad \times\left(\hat{\xi}_{\mathbf{k}}^{\prime}-\Delta \tau|\mathbf{k}|^{2}\left\{\hat{\phi}_{\mathbf{k}}^{n}-i \mathbf{k} \mathscr{F}_{\mathbf{k}}\left[\kappa_{0} \Omega^{*} \mathscr{F}_{\mathbf{k}^{\prime}}^{-1}\left[i \mathbf{k}^{\prime} \hat{\eta}_{\mathbf{k}^{\prime}}^{n}\right]\right]\right\}\right),
\end{aligned}
$$

где $\hat{\eta}_{\mathbf{k}}^{n}$ - фурье-образ параметра порядка в момент времени $\tau_{n}=n \Delta \tau, \mathscr{F}_{\mathbf{k}}[\cdot]$ и $\mathscr{F}_{\mathbf{k}^{\prime}}^{-1}[\cdot]-$ прямое и обратное преобразования Фурье соответственно, $\hat{\phi}_{\mathbf{k}}^{n}-$ фурьеобраз производной $\phi$ от плотности свободной энергии в момент времени $\tau_{n}, \mathbf{k}$ и $\mathbf{k}^{\prime}-$ волновые векторы, $\hat{\xi}_{\mathbf{k}^{\prime}}^{\prime}$ фурье-образ случайного поля $\xi^{\prime}\left(\mathbf{r}^{*}\right)=\int_{\tau}^{\tau+\Delta \tau} \xi^{*}\left(\mathbf{r}^{*}, \tau^{\prime}\right) d \tau^{\prime}$, которое с учетом (5) не зависит от времени $\tau$. Безразмерная константа $\tilde{\Omega}$, выбираемая из соображений устойчивости разностной схемы (6), была принята равной $\tilde{\Omega}=1$.

Для случайных величин $\xi_{\mathrm{k}}^{\prime}$ из (5) следует выражение для корреляционной функции (см. [20-23]):

$$
\left\langle\hat{\xi}_{\mathbf{k}}^{\prime} \hat{\xi}_{\mathbf{k}^{\prime}}^{\prime}\right\rangle=\Gamma(\mathbf{k}) \delta\left(\mathbf{k}+\mathbf{k}^{\prime}\right),
$$

где функция $\Gamma(\mathbf{k})$ определяется с помощью формулы:

$$
\Gamma(\mathbf{k})=\frac{8 \varepsilon \Delta \tau T^{*}}{N a^{d}}\left[3-\cos \left(k_{x} a\right)-\cos \left(k_{y} a\right)-\cos \left(k_{z} a\right)\right],
$$

$N$ - нормировочная константа преобразования Фурье. Непосредственное вычисление $\hat{\xi}_{\mathbf{k}}^{\prime}$ теперь может быть выполнено в виде: $\hat{\xi}_{\mathbf{k}}^{\prime}=a_{\mathbf{k}}+i b_{\mathbf{k}}$, где $a_{\mathbf{k}}$ и $b_{\mathbf{k}}-$ случайные величины, удовлетворяющие распределению Гаусса и характеризуемые рядом свойств [19,20,23]:

$$
\begin{gathered}
\left\langle a_{\mathbf{k}}\right\rangle=\left\langle b_{\mathbf{k}}\right\rangle=0, \quad\left\langle a_{\mathbf{k}}^{2}\right\rangle=\left\langle b_{\mathbf{k}}^{2}\right\rangle=\frac{1}{2} \Gamma(\mathbf{k}), \\
a_{\mathbf{k}}=a_{-\mathbf{k}}, \quad b_{\mathbf{k}}=-b_{-\mathbf{k}} .
\end{gathered}
$$

Все сплавы имеют атомную природу, что должно быть учтено введением максимального значения волнового вектора $k_{\max }$ (параметр обрезания) [22,23,25]. Данное значение подразумевает отсутствие волн концентрации вещества, имеющих длину меньшей, чем $\lambda<2 \pi / k_{\max }$. Данное условие может быть отражено с помощью условия, накладываемого на Фурье-компоненты случайного поля $\xi^{\prime}: \xi_{\mathbf{k}}^{\prime} \equiv 0$ при $|k|>k_{\max }[20,25,36]$. Выбор амплитуды и параметра обрезания флуктуаций (параметры $\varepsilon$ и $\left.k_{\max }\right)$ может существенно влиять на результаты моделирования процесса фазового перехода [20-23], в том числе на расчетные значения скоростей зарождения и роста выделений [23]. Данная особенность объясняется некоторым искажением фазового равновесия флуктуациями, которые могут привести к эффективному изменению критической температуры [37]. В дальнейшем моделирование будет проводиться при значениях параметров $\varepsilon=0.25, k_{\max }=1.2 a^{-1}$, рассмотренных в работе [23].

Идентификация выделений вторых фаз проводилась с помощью метода ближайшего соседа [38] в соответствии с разработанным авторами алгоритмом [34,39]. Пороговое значение усредненного состава, позволяющего отнести произвольный узел решетки ко второй фазе, принимался равным $C_{\min }=50 a t \%$. Размер выделения характеризовался эквивалентным радиусом $R[34,38]$. Концентрация выделений $X_{C}$ рассчитывалась как $X_{C}=N_{C} / V$, где $N_{C}$ - полное количество обнаруженных выделений, $V$ - объем, занимаемый рассматриваемой системой.

\section{4. Результаты моделирования}

На основе представленной модели и разностной схемы (6) был выполнен расчет распада трехмерного фрагмента сплава в форме куба, имеющего размер $L=256 a$, содержащего плоскую границу зерна с координатой $z=L / 2$. Моделирование проводилось для температуры $T^{*}=0.65$ и составов сплава $c_{M}=10$ и $11 \mathrm{at} \%$. Изменение взаимодействия на границе зерна описывалось значениями $\Delta=-0.25, \delta_{0}=2 a, \kappa_{0}=0.2$. Шаг по времени был принят равным $\Delta \tau=5.0 \pm 10^{-4}$.

Результаты моделирования эволюции концентрационного поля приведены на рис. 1. Как следует из рисунков, для случая $c_{M}=10 \mathrm{at} \%$ наблюдается режим преимущественной зернограничной преципитации $[17,18]$, что соответствует частичному смачиванию границы зерна [1,14-16]. Небольшие зародыши, формирующиеся вдали от границы зерна, являются неустойчивыми и неизбежно растворяются, что, по всей видимости, связано с большим значением барьера нуклеации, наблюдаемым при низких степенях пересыщения [5,6]. Для случая бо́льшей концентрации $c_{M}=11 \mathrm{at} \%$ наблюдается смешанный режим распада (см. также $[17,18])$, для которого образование выделений происходит как в объеме зерна, так и на его границе.

В обоих рассмотренных случаях выделения второй фазы на границе имеют линзовидную (пластинчатую) форму, а в объеме зерна - форму, близкую к сферической. 

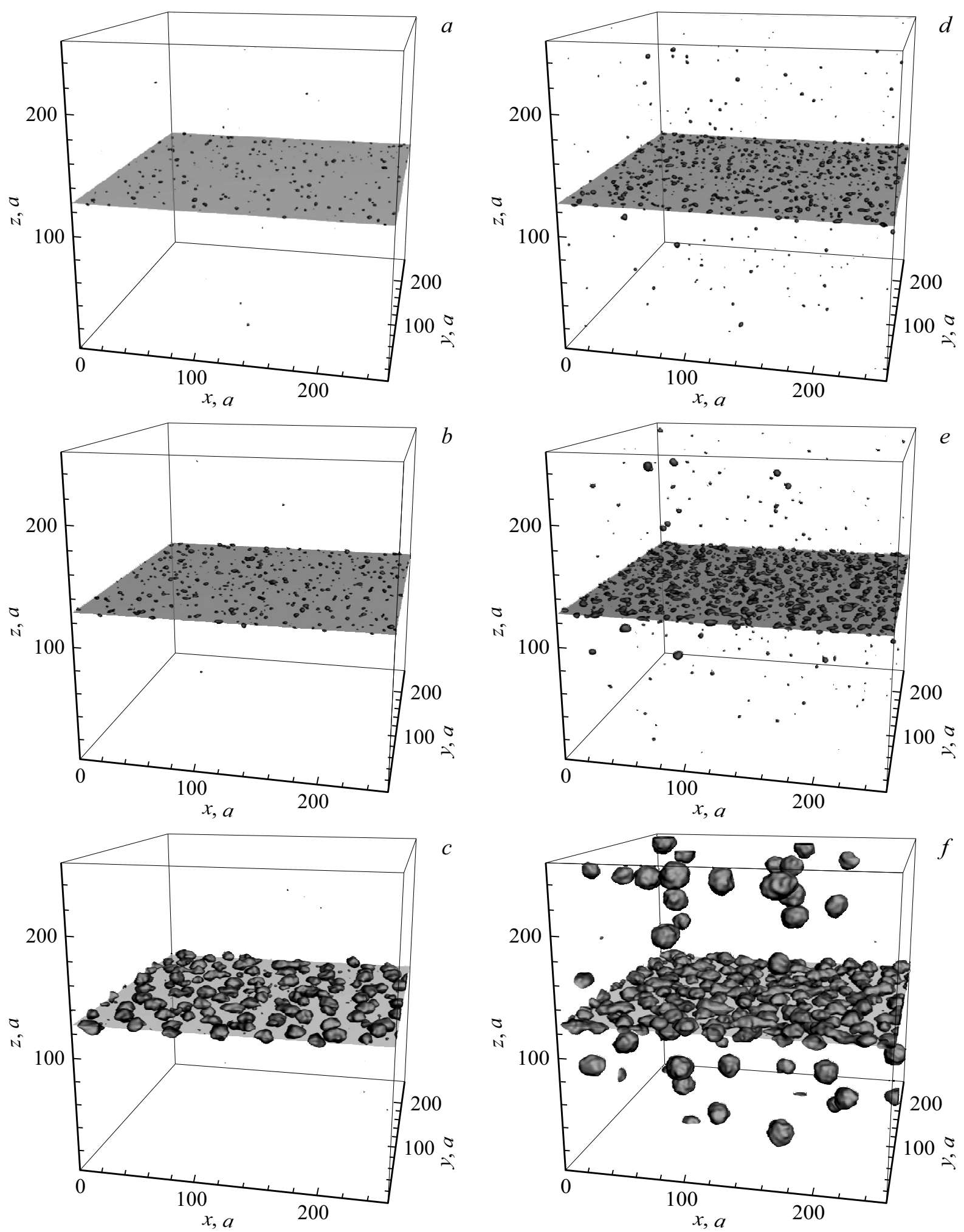

Рис. 1. Распределения фаз в бинарном сплаве при температуре $T^{*}=0.65$, имеющим составы $c_{M}=10$ at $\%$ (рис. $\left.a, b, c\right)$ и $c_{M}=11$ at\% (pис. $d, e, f$ ) в различные моменты времени: $\tau=75$ (рис. $\left.a, d\right), \tau=150$ (рис. $\left.b, f\right), \tau=900$ (pис. $c, f$ ).

Наблюдаемая геометрия выделений (рис. 1) на границе и в объеме зерна согласуется с результатами экспериментальных исследований смачивающих фазовых переходов на границах зерен для ряда сплавов [14-16].
Наличие в системе длинноволновых флуктуаций приводят к возникновению рельефности поверхности выделений, расположенных как в объеме зерна (см. также, [23]), так и на его границе. Флуктуации также 
обуславливают непостоянство контактного угла, который может изменяться в процессе роста в достаточно широких пределах. При анализе контактных углов крупных выделений, образовавшихся на границах зерен рассматриваемых сплавов на поздней стадии, рассчитанные углы составили: $52^{\circ} \pm 10^{\circ}$ для $c_{M}=11 \mathrm{at} \%$ и $56^{\circ} \pm 11^{\circ}$ для $c_{M}=10 \mathrm{at} \%$. Совпадение полученных контактных углов в пределах ошибки является вполне ожидаемым и обусловлено одинаковыми значениями температуры, а также параметрами взаимодействия для обоих сплавов.

Для изучения кинетики среднего размера, концентрации, функции распределения выделений на ранней стадии $(\tau<275)$ было проведено независимое моделирование для четырех фрагментов твердого раствора с помощью разностной схемы (6). Характеристики распределения фаз для данного интервала определялись путем их усреднения по всем независимым реализациям $[18,40]$. Для более поздних интервалов времени $(\tau>275)$ проводилась одна попытка расчета ввиду существенной длительности расчетов. Длительность расчетов на рассматриваемом интервале $0<\tau<1600$ составила около четырех суток.

Результаты расчета кинетики среднего размера выделений и их концентрации приведены на рис. 2 и 3. Как следует из рисунков, для рассмотренных составов и интервалов времени для сплава наблюдаются известные механизмы формирования выделений вторых фаз (зарождение, рост, коагуляция, коалесценция). Вместе с тем скорость роста среднего размера выделений существенно отличается от известных зависимостей, установленных в классической теории нуклеации $[5,41]$ для стадий диффузионного роста $\left(\langle R\rangle \sim \tau^{1 / 2}\right)$ и коалесценции $\left(\langle R\rangle \sim \tau^{1 / 3}\right)$. Данные зависимости достаточно хорошо подтверждаются на основе моделирования нуклеации в бинарных сплавах с постоянной подвижностью, не имеющих структурных дефектов, в отсутствие флуктуаций состава $[34,42]$.

На стадии зарождения в обоих рассмотренных случаях наблюдался рост среднего размера, близкий к степенному закону. При этом стадия диффузионного роста характеризовалась ускоренным ростом среднего размера выделений по сравнению с классической теорией диффузионного роста, в соответствии с которой радиус выделения должен изменяться как $R \sim \tau^{1 / 2}[5,41]$. Причиной аномального степенного роста на ранней стадии является комбинация механизмов распада, наблюдаемая при рассматриваемых малых концентрациях растворенного компонента. Так, на ранней стадии происходит образование зародышей в течение достаточно продолжительного временного интервала. Устойчивые зародыши, образовавшиеся ранее, испытывают диффузионный рост за счет притока атомов из матрицы. Одновременно происходит формирование новых зародышей, при этом эффективная скорость роста среднего размера оказывается ниже, чем предполагает теория диффузионного роста $\langle R\rangle \sim \tau^{\alpha}$ $(\alpha<1 / 2)$. Затем, по мере уменьшения степени пересыщения, зародыши, образовавшиеся позднее, оказываются

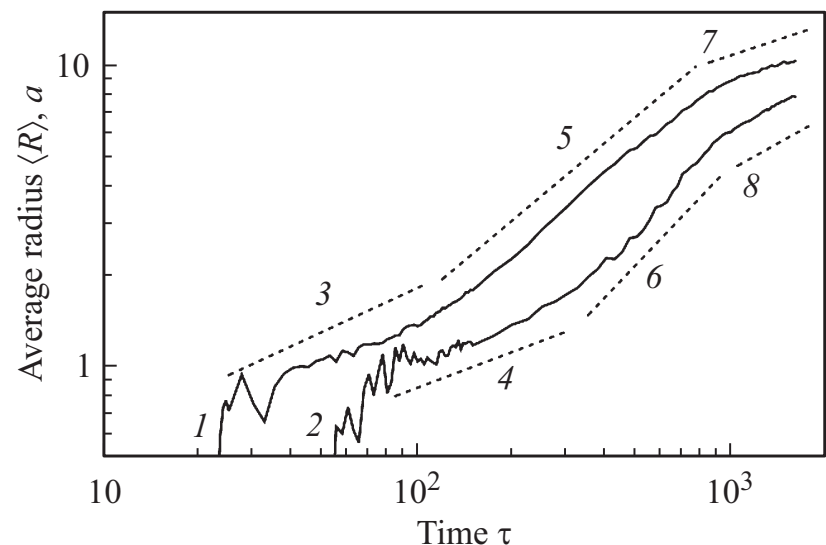

Рис. 2. Зависимость среднего размера выделений $\langle R\rangle$ в бинарном сплаве от времени отжига $\tau$ для сплавов $c_{M}=11 \mathrm{at} \%$ (кривая 1 ) и $c_{M}=10 \mathrm{at} \%$ (кривая 2). Пунктирными линиями приведена аппроксимация соответствующих расчетных данных степенными функциями $\langle R\rangle \propto \tau^{\alpha}: 3-\alpha=0.48 \pm 0.02,4-\alpha=0.39 \pm 0.03,5-$ $\alpha=0.87 \pm 0.01,6-\alpha=1.1 \pm 0.03,7-\alpha=0.34 \pm 0.01$, $8-\alpha=0.59 \pm 0.01$.

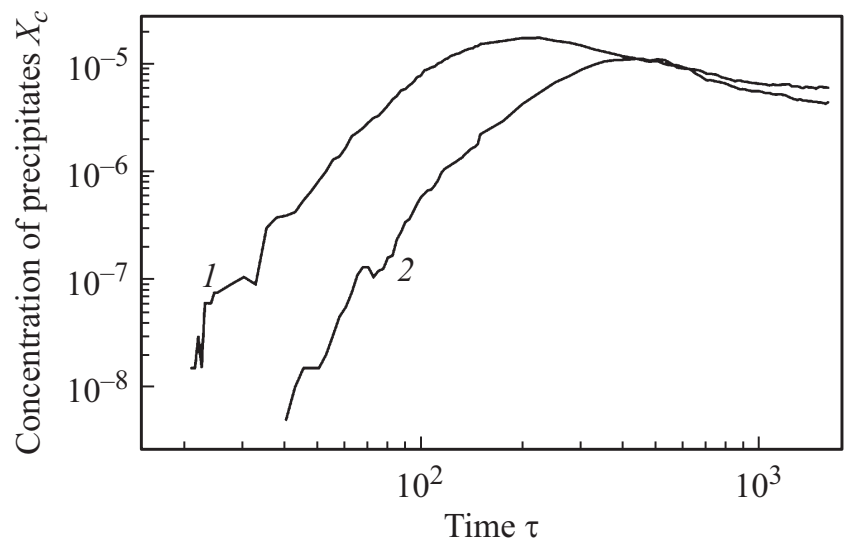

Рис. 3. Зависимость концентрации выделений $X_{C}$ от времени отжига $\tau$ для сплавов с составами $c_{M}=11$ at\% (кривая 1 ) и $c_{M}=10$ at\% (кривая 2) при температуре $T^{*}=0.65$.

в неустойчивом состоянии и быстро растворяются. Данный процесс сочетается с продолжающимся диффузионным ростом устойчивых зародышей, что приводит к эффективному росту среднего размера $\langle R\rangle \sim \tau^{\alpha}(\alpha>1 / 2)$. Наличие границ зерен также обуславливает различную скорость протекания процессов формирования и роста выделений в объеме и на границе зерна, которые фактически находятся на разных стадиях распада, что и приводит к изменению показателя степени $\alpha$.

К концу рассмотренного интервала $(\tau>1000)$ наблюдается существенное замедление скорости роста, что может быть связано с постепенным переходом к стадии коалесценции. Данная промежуточная стадия (см. также $[34,40,42])$ характеризуется слабым ростом среднего размера при медленном уменьшении концентрации выделений. Ожидается, что промежуточная стадия 


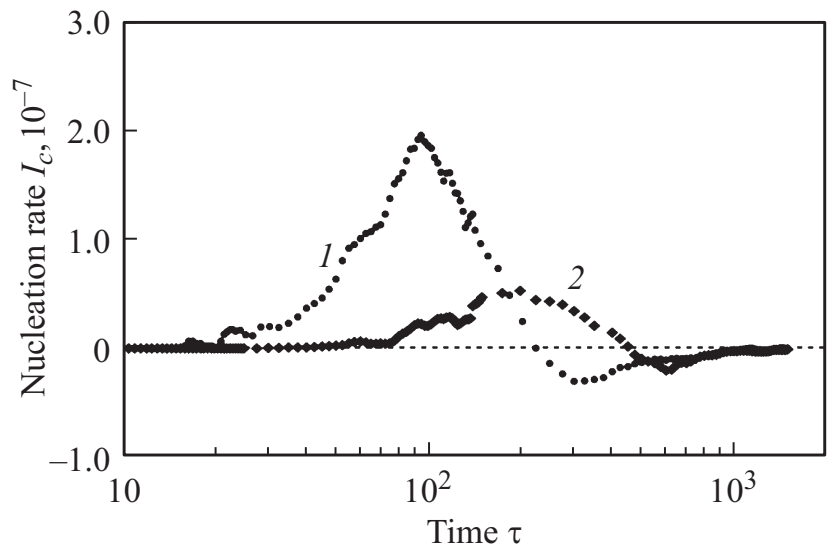

Рис. 4. Зависимость скорости зарождения $I_{C}$ выделений второй фазы в бинарных сплавах, характеризуемых составами $c_{M}=10$ at $\%$ (кривая 2) и $c_{M}=11$ at $\%$ (кривая 1) при температуре $T^{*}=0.65$.

может оказаться существенно более длительной, чем для сплава без границы зерна $[34,42]$, что связано с комбинацией нескольких механизмов: коагуляции (слияния) выделений, находящихся на границе зерна, а также диффузионного роста этих выделений за счет вещества, поступающего из матрицы. На данном этапе также наблюдается и растворение мелких выделений, свойственное механизму коалесценции, однако данный механизм не является доминирующим. Кроме того, существенное влияние на процесс переноса вещества и рост выделений оказывают флуктуации (см. также [23]). Изучение роста выделений второй фазы на поздней стадии требует увеличения вычислительной мощности или времени моделирования примерно на один-два порядка, а также существенного увеличения размера системы.

На основе рассмотренной модели могут быть установлены также и некоторые особенности кинетики формирования выделений второй фазы. Скорость зарождения выделений может быть определена как производная от концентрации наблюдаемых выделений $I_{C}=d X_{C} / d \tau$ [43]. В классической и неклассической теориях нуклеации данная величина обычно связывается только с закритическими зародышами $[5,41,43]$. В рассматриваемом случае параметры взаимодействия зависят от координат, а изменение состава сплава по объему системы на стадии зарождения определяется флуктуациями. При этом не представляется возможным отличить закритические зародыши от других зародышей и неоднородностей состава, сформированных в системе благодаря флуктуациям $[23,34,43]$. В связи с этим величина $I_{C}$ фактически является измеряемой скоростью зарождения („detectable nucleation rate“ [43]), значение которой может зависеть от использованного метода идентификации зародышей.
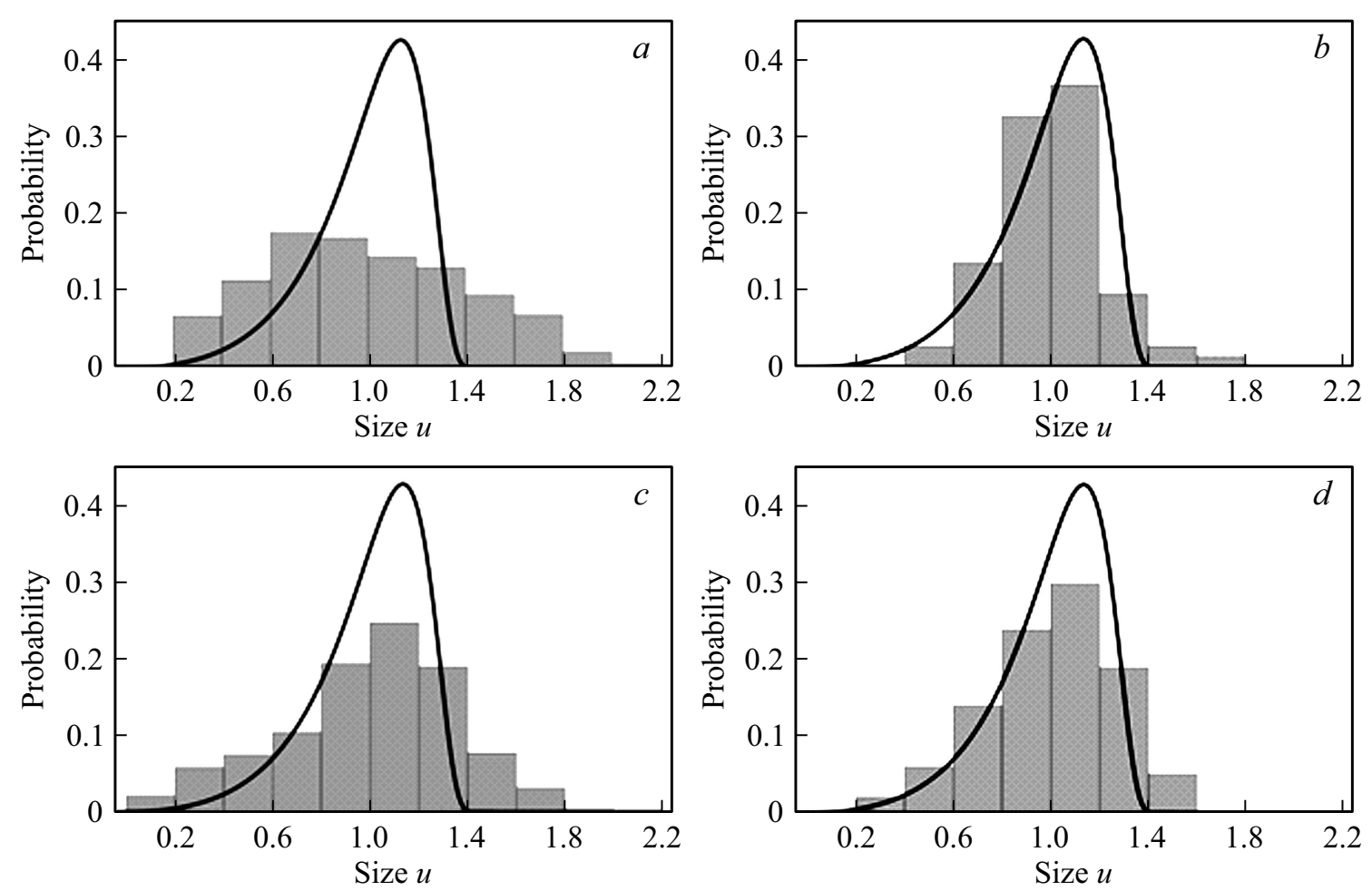

Рис. 5. Функция распределения по размерам $(u=R /\langle R\rangle)$ в случае формирования выделений на границах зерен для сплавов, имеющих состав $c_{M}=10 \mathrm{at} \%(a, b)$ и $c_{M}=11 \mathrm{at} \%(c, d)$, в различные моменты времени $\tau=400(a, c)$ и $\tau=1600(b, d)$ при температуре $T^{*}=0.65$. Сплошной линией отражена функция распределения по размерам в соответствии с теорией Лифшица-Слезова. 
На рис. 4 представлена зависимость скорости зарождения для двух рассмотренных составов сплава. Поскольку рассматриваемая величина имеет существенную случайную составляющую, при построении данных кривых было использовано сглаживание по методу скользящей средней [44]. Как следует из рисунка, уменьшение состава сплава приводит к снижению скорости зарождения, а также к заметному увеличению инкубационного времени зародышеобразования. Данная особенность хорошо согласуется с выводами неклассической теории нуклеации $[5,6]$, в которой установлено увеличение критического размера и инкубационного времени при уменьшении степени пересыщения (см. например $[34,45,46]$ ).

Рассматриваемый подход позволяет также проследить эволюцию функции распределения по размерам, результат расчета которой для рассматриваемых сплавов $\left(c_{M}=10\right.$ и 11 at $\left.\%\right)$ представлен на рис. 5. Как следует из рисунков, функции распределения для рассматриваемых сплавов проходят эволюцию, сходную со сплавами, не имеющими структурных дефектов $[23,34,42]$. На ранней стадии зародыши имеют достаточно большой разброс по наблюдаемым размерам, максимальное значение $R$ может более, чем в два раза $\left(u_{\max }>2.0\right)$ превышать $\langle R\rangle$ (рис. 5, $a$ и $c$ ). На поздней стадии функция распределения приближается к функции, определяемой теорией Лифшица-Слезова $\left(u_{\max } \sim 3 / 2\right)$. Формирование стационарной функции распределения по размерам для сплава с меньшим составом заметно запаздывает (рис. 5, $b$ и $c$ ). Необходимо отметить, что вид стационарной функции распределения по размерам на поздней стадии распада существенно зависит от механизма переноса вещества [47]. В общем случае сочетание нескольких механизмов или доминирование одного из них (например, зернограничной диффузии) может приводить к функциям распределения, существенно отличающимся от выводов теории Лифшица-Слезова, построенной для случая объемной диффузии растворенного компонента [47]. В системах, характеризуемых различными механизмами массопереноса, ожидается возникновение стационарных функций распределения выделений с различными значениями параметра $u_{\max }$. Несмотря на существенно разные условия формирования выделений, принципиальных различий между функциями распределения для рассмотренных случаев зернограничной преципитации $\left(c_{M}=10 \mathrm{at} \%\right)$ и смешанного режима распада $\left(c_{M}=11\right.$ at $\left.\%\right)$ не обнаружено, что, по всей видимости, связано с доминированием переноса вещества по объему системы. Возможной причиной слабого различия функций распределения является также недостаточность рассмотренного интервала времени, на котором стационарная функция распределения могла быть недостигнута.

\section{5. Заключение}

На основе разработанной модели и проведенного моделирования могут быть сделаны следующие выводы о процессе преципитации на границах зерен, который может наблюдаться, если параметр взаимодействия между компонентами раствора уменьшается при приближении к границе зерна.

Наиболее выраженное влияние границ зерен на процесс преципитации наблюдается в случае малой степени пересыщения. В этом случае образование зародышей происходит непосредственно на границе зерна, а в объеме зерна выделения не наблюдаются. Основной причиной является снижение барьера нуклеации вблизи границ зерен, обусловленное более низким значением параметра взаимодействия. Преодоление барьера нуклеации обеспечивается за счет длинноволновых флуктуаций состава сплава.

При повышении концентрации растворенного компонента сплав переходит к смешанному механизму формирования выделений, когда устойчивые зародыши образуются как в объеме, так и на границе зерна. Повышение концентрации растворенного компонента приводит к росту скорости флуктуационного зарождения и сокращению инкубационного времени. Продолжительность стадий зарождения и диффузионного роста при этом сокращается.

Флуктуации приводят к возникновению рельефности поверхности выделений, что существенно влияет на определяемые значения контактных углов. По мере роста выделений контактный угол может изменяться в достаточно широких пределах. Для крупных выделений, формирующихся на поздней стадии распада, изменчивость контактного угла снижается.

Флуктуации и зависимость параметров взаимодействия от координат, связанная с наличием границы зерна, приводят к возникновению некоторых особенностей роста выделений. На ранней стадии может быть выделен участок слабого степенного роста выделений $\left(\langle R\rangle \propto \tau^{\alpha}\right.$, $\alpha \sim 0.3)$. На стадии диффузионного роста наличие границ зерен и флуктуаций состава может приводить к возникновению ускоренного роста выделений $(\alpha>1 / 2)$. В случае преимушественной зернограничной преципитации может наблюдаться существенно замедленный переход к стадии коалесценции. К возникновению ускоренного роста мог привести комплекс причин, связанных с рассматриваемой системой. Во-первых, наличие флуктуаций состава приводит к одновременному наблюдению нескольких механизмов формирования второй фазы: зарождения, диффузионного роста и растворения выделений, а также их слияния (коагуляции). Данное сочетание различных механизмов формирования второй фазы может существенно влиять на скорость роста среднего размера выделений вторых фаз. Во-вторых, уменьшение энергии взаимодействия вблизи границы зерна приводит к различию степеней пересыщения в объеме и на границе зерна, при этом эффективный коэффициент диффузии вблизи границ зерен увеличивается, что обуславливает различие потоков вещества вдоль границы зерна и из объема зерна к границе. Кроме того, ввиду различия скорости формирования и 
роста вторых фаз на границе и в объеме зерна распад в данных областях может находиться на разных стадиях, что также обуславливает изменение скорости роста усредненных по всей рассматриваемой системе значений размера и концентрации выделений.

\section{Список литературы}

[1] Б.Б. Страумал. Фазовые переходы на границах зерен. Наука, М. (2003). 327 с.

[2] Физическое металловедение. Т. 2. / Под. ред. Р.У. Кана, П. Хаазена. Металлургия, М. (1987). 624 с.

[3] L. Priester. Grain Boundaries: From Theory to Engineering. Springer Dordrecht. (2013). 441 p.

[4] P. Lejček, M. Všianská, M. Šob. J. Mater. Res. 332647 (2018).

[5] K. Kelton, A. Greer. Nucleation in condensed matter. Elsevier (2010). $726 \mathrm{p}$.

[6] J.W. Cahn, J.E. Hilliard. J. Chem. Phys. 31, 688 (1959).

[7] J. Cahn. Acta Metall. 9, 795 (1961).

[8] N. Provatas, K. Elder. Phase-field methods in material science and engineering. Wiley-WCH, Weinheim. (2010). 298 p.

[9] J. Cahn. J. Chem. Phys. 66, 3667 (1977).

[10] D. Bonn, J. Eggers, J. Indekeu, J. Meunier, E. Rolley. Rev. Mod. Phys. 81, 739 (2009).

[11] A.A. Pahlavan, L. Cueto-Felgueroso, A.E. Hosoi, G.H. McKinley, R. Juanes. J. Fluid Mech. 845, 642 (2018).

[12] K. Mahdy, A. Afkhami, L. Kondic. Phys. Fluids 26, 62002 (2016).

[13] G. Grün, K. Mecke, M. Rauscher. J. Status. Phys. 122, 1261 (2006).

[14] О.А. Когтенкова, А.Б. Страумал, Н.С. Афоникова, А.А. Мазилкин, К.И. Колесникова, Б.Б. Страумал. ФТТ 58, 721 (2016).

[15] A.S. Gornakova, B.B. Straumal, A.N. Nekrasov, A. Kilmametov, N.S. Afonikova. J. Mater. Eng. Perform. (2018); DOI: $10.1007 / \mathrm{s} 11665-018-3300-3$.

[16] B.B. Straumal, O.A. Kogtenkova, M.Yu. Murashkin, M.F. Bulatov, T. Czeppe, P. Zięba. Mater. Lett. 186, 82 (2017).

[17] П.Е. Львов, В.В. Светухин. ФТТ 59, 2425 (2017).

[18] П.Е. Львов, В.В. Светухин ФТТ 60, 787 (2018).

[19] J. Fan, M. Sammalkorpi, M. Haataja. Phys. Rev. E. 81, 11908 (2010).

[20] T.M. Rogers, K.R. Elder, R.C. Desai. Phys. Rev. B 37, 9638 (1988).

[21] K.R. Elder, T.M. Rogers, R.C. Desai. Phys. Rev. B 38, 4725 (1988).

[22] J. Garsía-Ojalvo, J.M. Sancho. Noise in spatially extended systems. Springer, N.Y. (1999). 314 p.

[23] P.E. L'vov, V.V. Svetukhin. Modelling Simul. Mater. Sci. Eng. 26, 045001 (2018).

[24] H. Cook. Acta Mater. 18, 297 (1970).

[25] P.C. Hohenberg, B.I. Halperin. Rev. Mod. Phys. 49, 435 (1977).

[26] X. Li, G. Ji, H. Zhang. J. Comp. Phys. 283, 81 (2015).

[27] В.Б. Федосеев. ФТТ 57, 585 (2015).

[28] В.Б. Федосеев, Е.Н. Федосеева. Письма в ЖЭТФ 97, 473 (2013).

[29] J.C. Fisher. J. Appl. Phys. 22, 74 (1951).

[30] Y. Mishin, Chr. Herzig, J. Bernardini, W. Gust. Int. Mater. Rev. 42, 155 (1997).
[31] В.В. Попов, А.В. Сергеев, Н.К. Архипова, А.Ю. Истомина. ФMM 112, 273 (2011).

[32] R.T. Sibatov, V.V. Svetukhin. Phys. Lett. A 381, 2021 (2017).

[33] J. Zhu, L.-Q. Chen, J. Shen, V. Takare. Phys. Rev. E 60, 3564 (1999).

[34] P.E. L'vov, V.V. Svetukhin. Mod. Simul. Mater. Sci. Eng. 25, 075006 (2017).

[35] L.-Q. Chen, J. Shen. Comp. Phys. Commun. 108, 147 (1998).

[36] S. Muralidharan, R. Khodadad, E. Sullivan, M. Haataja. Phys. Rev. B 85, 245428 (2012).

[37] Л.Д. Ландау, Е.М. Лифшиц. Статистическая физика. Наука, М. Ч. 1 (1976), 584c.

[38] M. Miller, R. Forbes. Atom-probe tomography: the local electrode atom probe. Springer, N.Y. (2014). 423 p.

[39] П.Е. Львов, В.В. Светухин. Свидетельство о государственной регистрации программы для ЭВМ. № 2017616554 от 08.07.2017.

[40] П.Е. Львов, В.В. Светухин. ФТТ 58, 1382 (2016).

[41] H. Aaronson, M. Enomoto, J. Lee. Mechanisms of Diffusional Phase Transformations in Metals and Alloys. CRC Press, Taylor \& Francis Group, Boca Raton (2010). 667 p.

[42] П.Е. Львов, В.В. Светухин. ФТТ 59, 345 (2017).

[43] D. Kashchiev. Nucleation. Basic Theory with Applications. Butterworth-Heinemann, Eastbourne. (2000). 551 p.

[44] Г.С. Кильдишев, А.А. Френкель. Анализ временных рядов и прогнозирование. Статистика, М. (1973). 103 с.

[45] F.K. LeGoues, H.I. Aaronson. Acta Met. 32, 1855 (1984).

[46] B. Li, L. Zhang, C. Li, Q. Li, J. Chen, G. Shu, Y. Weng, B. Xu, W. Liu. Mod. Simul. Mater. Sci. Eng. 25, 085006 (2017).

[47] В.В. Слезов, В.В. Сагалович. УФН 151, 67 (1987).

Редактор Т.Н. Василевская 